\title{
Microwave resonance of the reentrant insulating quantum Hall phases in the 1st excited Landau Level
}

\author{
R. M. Lewis ${ }^{1,2}$, Yong P. Chen ${ }^{1,2}$, L. W. Engel ${ }^{1}$, D. C. Tsui ${ }^{2}$, L. N. Pfeiffer ${ }^{3}$, and K. W. West ${ }^{3}$ \\ ${ }^{1}$ NHMFL, Florida State University, Tallahassee, FL 32310, USA \\ ${ }^{2}$ Dept. of Electrical Engineering, Princeton University, Princeton, NJ 08544 \\ ${ }^{3}$ Bell Laboratories, Lucent Technologies, Murray Hill, NJ 07974
}

(Dated: November 25, 2018)

\begin{abstract}
We present measurements of the real diagonal microwave conductivity of the reentrant insulating quantum Hall phases in the first excited Landau level at temperatures below $50 \mathrm{mK}$. A resonance is detected around filling factor $\nu=2.58$ and weaker frequency dependence is seen at $\nu=2.42$ and 2.28. These measurements are consistent with the formation of a bubble phase crystal centered around these $\nu$ at very low temperatures.
\end{abstract}

Clean two-dimensional electron systems (2DES) show a plethora of phases at low temperatures $(T)$ when subjected to perpendicular magnetic fields $(B)$. The most well known of these are the integer [1] and fractional 2] quantum Hall effects (IQHE and FQHE). Both are identified by quantized Hall resistances, $R_{x y}=h / \nu e^{2}$ and the simultaneous vanishing of the diagonal resistance $\left(R_{x x}\right)$ when the filling factor $\nu=n h / e B$ coincides with a gap in the single or many-particle density of states. In higher Landau levels (LL), $N \geq 2$, where $N$ is the LL index, vanishing minima in $R_{x x}$ are observed [3, 4] near $\nu=4+1 / 4,4+3 / 4$, and several subsequent odd quarter $\nu$. However, at these $\nu, R_{x y}$ is quantized to the value of the adjacent integer plateau. Therefore these states are said to exhibit a reentrant integer quantum Hall effect (RIQHE) [5]. Theory [6, 7, [8, [9] predicts a crystal phase of the 2DES with triangular lattice symmetry and two or more electrons per lattice site - pinned by disorder and hence insulating - to occur at approximately these $\nu$. This crystal has been dubbed the bubble phase (BP). Experiments have subsequently shown nonlinear current-voltage characteristics 5, a microwave resonance [10], and narrow band noise [11], in the range of $\nu$ centered around $\nu=4+1 / 4$. Still more recent work 12 has looked at the transition between the BP around $\nu=4+1 / 4$ and another solid phase - presumably a Wigner crystal [13, 14, 15] of carriers in the uppermost LL-close to $\nu=4$.

The microwave measurements 10, 12], which are similar to those we will discuss here, provide compelling evidence that the RIQHE is due to the formation of a pinned electron solid such as the BP. These measurements detect a sharp resonance in the real diagonal conductivity $\left(\operatorname{Re}\left[\sigma_{x x}(f)\right]\right)$ when the $2 \mathrm{DES}$ forms an electron solid. The observed resonant frequency, $f_{p k}$, is considerably lower, in terms of energy, than the temperature at which the resonance first appears, $f_{p k}<<k_{B} T / h$, indicating that the resonance is not due to the ionization of individual carriers trapped in potential defects. Furthermore, this resonance is strikingly similar to the resonance which occurs in the insulating phase of $2 \mathrm{DES}[16,17$ that terminates the FQHE at high $B, \nu<1 / 5$, where the 2 DES is thought to form a Wigner crystal[15]. The resonances in both regimes (RIQHE and $\nu<1 / 5$ insulating phase) are interpreted as pinning modes 18, 19] of crystal domains oscillating within the disorder potential.

Recently, Eisenstein et al. 20] and Xia et al. 21] have presented measurements of RIQHE's in the $N=1$ LL which appear only at $T \lesssim 50 \mathrm{mK}$. These RIQHE's are centered at partial filling factors $\nu^{*} \approx 0.28,0.42$, 0.56 , and 0.70 where $\nu^{*}=\nu-[\nu]$ and $[\nu]$ is the greatest integer less than $\nu$. The initial theories [6, 7] of charge density waves in higher LL (which predicted the stripe and $\mathrm{BP}$ for $\nu>4$ ) did not consider the $\mathrm{N}=1 \mathrm{LL}$. Also, the short range softening of the effective electron-electron potential, which gives rise to the BP in higher LL, is only weakly present in the $\mathrm{N}=1 \mathrm{LL}$ and absent in the $\mathrm{N}=0 \mathrm{LL}$. However, more recent theories 22, 23, 24] do predict a BP and perhaps a stripe phase in the first excited LL.

In this paper, we present measurements of $\operatorname{Re}\left[\sigma_{x x}\right]$ between $\nu=2$ and 3 . At $T \approx 35 \mathrm{mK}$, we show that a resonance occurs in the frequency $(f)$ dependence of $\operatorname{Re}\left[\sigma_{x x}\right]$ in a range of $\nu$ around 2.58. An enhancement of $\operatorname{Re}\left[\sigma_{x x}\right]$ at $\nu \approx 2.42$ is also observed at low $f$. These features are not present for $T \geq 55 \mathrm{mK}$. The resonance occurs at frequency, $f \sim 125 \mathrm{MHz}$, and is coincident with the RIQHE phase found previously [20, 21] in the $\mathrm{N}=1 \mathrm{LL}$ on either side of $\nu=5 / 2$. In light of our earlier observations 10 . in higher LL's, this resonance is naturally interpreted as due to the pinning mode of the $\mathrm{BP}$ in the $\mathrm{N}=1 \mathrm{LL}$ at $\nu=2.57$.

The sample, grown by molecular beam epitaxy, is a $300 \AA \mathrm{GaAs} / \mathrm{AlGaAs}$ quantum well of density $\mathrm{n}=3.0 \times 10^{11} \mathrm{~cm}^{-2}$ and mobility $\mu=2.4 \times$ $10^{7} \mathrm{~cm}^{2} \mathrm{~V}^{-1} \mathrm{~s}^{-1}$ at $300 \mathrm{mK}$. The quantum well is approximately $2000 \AA$ below the surface. A coplanar waveguide [25] (CPW) is patterned onto the sample surface. The CPW consists of a driven centerline of length $l$ which is separated from symmetrically placed ground planes by slots of width $w$. At high $f$ and small $\left|\sigma_{x x}\right|$, the conductivity of the 2DES attenuates power transmission along the $\mathrm{CPW}$ and $\operatorname{Re}\left[\sigma_{x x}\right]=-\frac{w}{2 l Z_{0}} \ln \left(P_{t} / P_{0}\right)$, where $P_{t}$ is the transmitted power 26]. In the absence of 2DES, the CPW has impedance $Z_{0}=50 \Omega$ and transmits power $P_{0}$. 


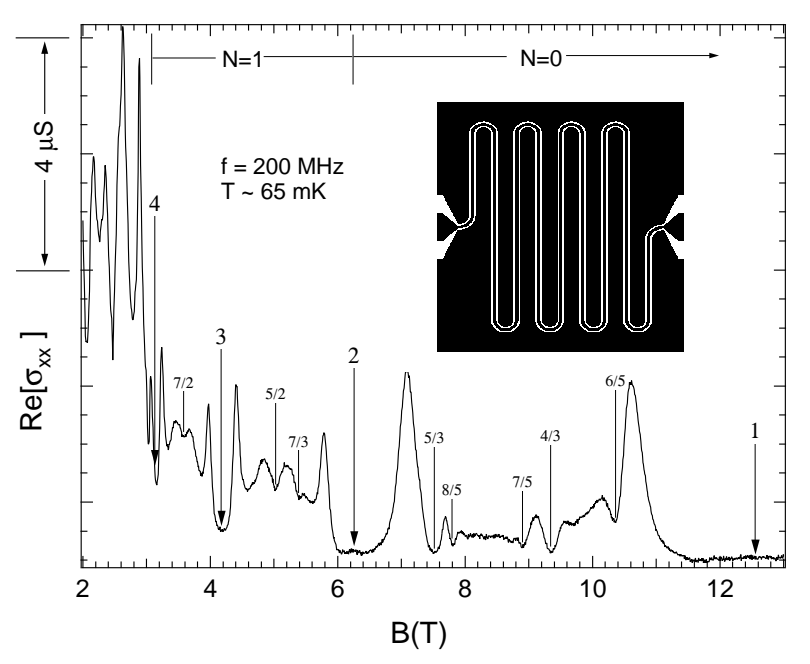

FIG. 1: The real part of the diagonal conductivity, $\operatorname{Re}\left[\sigma_{x x}\right]$ versus $B$ between 2 and $13 \mathrm{~T}$. Inset is the coplanar waveguide pattern in which dark regions are the metal films.

The CPW pattern is shown in the inset to Fig. 1 and has $l=28 \mathrm{~mm}$ and $w=30 \mu \mathrm{m}$. The dark regions are the metal films of the CPW. Measurements were carried out in the low power limit which was determined by reducing microwave power until no further change in $\operatorname{Re}\left[\sigma_{x x}\right]$ was observed.

In Fig. 1 we show $\operatorname{Re}\left[\sigma_{x x}\right]$ measured, between 2 and $13 \mathrm{~T}$ at $200 \mathrm{MHz}$ and $T \approx 65 \mathrm{mK}$. The broad minima centered around $B=12.55,6.26,4.18$, and $3.14 \mathrm{~T}$ are due to the IQHE states at $\nu=1,2,3$, and 4 . Between $\nu=1$ and 2, sharp minima indicate FQHE states in the $\mathrm{N}=0$ LL at $\nu=6 / 5,4 / 3,7 / 5,8 / 5$, and $5 / 3$. The $\mathrm{N}=1$ LL also shows FQHE states. For instance, at $B=5.38$, 5.03 , and $3.58 \mathrm{~T}$, dips due to the $\nu=7 / 3$, the $5 / 2$, and $7 / 2$ FQHE are apparent. For $\nu>4(\mathrm{~N}=2 \mathrm{LL})$, sharp peaks appear in $\operatorname{Re}\left[\sigma_{x x}\right]$ at $B \approx 2.89,2.63,2.36$, and $2.17 \mathrm{~T}$, or roughly at $\nu \approx 4+1 / 4,4+3 / 4,5+1 / 4$, and $5+3 / 4$ where DC measurements [3, 4, 5] observe the RIQHE. These peaks are due to a resonance at $f \approx 300$ $\mathrm{MHz}$, which was interpreted [10] as due to the pinning mode 18 of the BP.

In Fig. 2, we focus on the range of $B$ between 4.2 and $6.2 \mathrm{~T}$ where $\nu$ lies within the $\mathrm{N}=1$ spin-up LL. In Fig. 2a, we show $\operatorname{Re}\left[\sigma_{x x}\right]$ at $f=125 \mathrm{MHz}$ for $T$ approximately at $30,40,50$ and $55 \mathrm{mK}$. The most striking aspects of these data are the dramatic increases of the peaks in $\operatorname{Re}\left[\sigma_{x x}\right]$ at $4.88 \mathrm{~T}$ (marked as P1) and at $5.17 \mathrm{~T}$ (marked as P2) with decreasing $T$. P1 occurs at $\nu=2.57$ and $\mathrm{P} 2$ at $\nu=2.42$. At $\mathrm{P} 1, \operatorname{Re}\left[\sigma_{x x}\right]$ increases by $\sim 4 \mu \mathrm{S}$ as $T$ decreases from $55 \mathrm{mK}$ to $30 \mathrm{mK}$. Similarly, at $\mathrm{P} 2, \operatorname{Re}\left[\sigma_{x x}\right]$ increases by about $1 \mu \mathrm{S}$ over the same $T$ range. Some $T$ dependence is also evident in the peak at $B=5.47 \mathrm{~T}(\nu=2.28)$, labelled as P3.

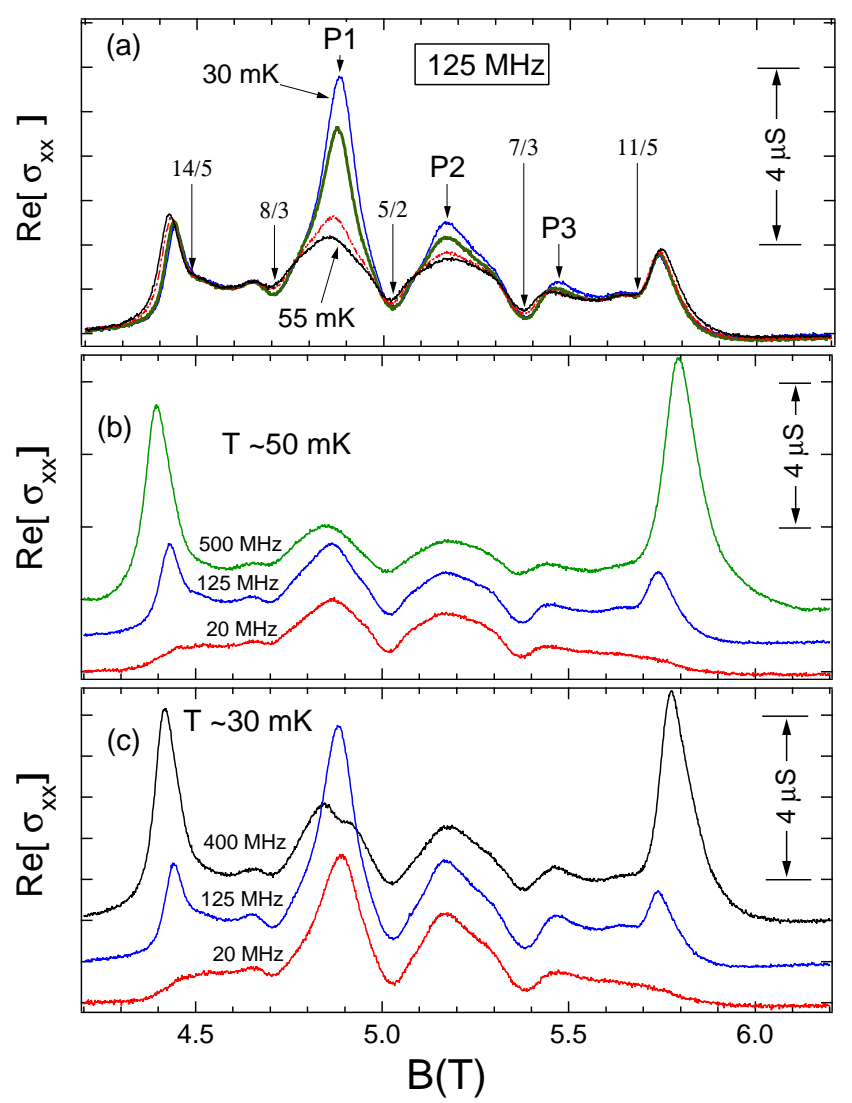

FIG. 2: (a) $\operatorname{Re}\left[\sigma_{x x}\right]$ vs $B$ between 4.2 and $6.2 \mathrm{~T}$ at frequency $f=125 \mathrm{MHz}$ for temperatures $T \approx 30,40,50$, and $55 \mathrm{mK}$. (b) $\operatorname{Re}\left[\sigma_{x x}\right]$ vs $B$ at $f=20,125$, and $500 \mathrm{MHz}$ with $T \approx 50$ mK offset $1 \mu \mathrm{S}$ from each other. (c), $\operatorname{Re}\left[\sigma_{x x}\right]$ vs $B$ at $f=20$, 125 , and $400 \mathrm{MHz}$, all at $T=30 \mathrm{mK}$ offset for clarity.

Fig. $2 \mathrm{~b}$ shows $\operatorname{Re}\left[\sigma_{x x}\right]$ vs $B$ measured at $f=20,125$, and $500 \mathrm{MHz}$ at $T \approx 50 \mathrm{mK}$. At this $T, \mathrm{P} 1$ and $\mathrm{P} 2$, show little dependence on $f$. The traces are offset $1 \mu \mathrm{S}$ from each other.

In Fig. 2c, we plot $\operatorname{Re}\left[\sigma_{x x}\right]$ vs $B$ for $f=20,125$, and $400 \mathrm{MHz}$, at $T \approx 30 \mathrm{mK}$ offset by $1 \mu \mathrm{S}$ each for clarity. At $\mathrm{P} 1, \operatorname{Re}\left[\sigma_{x x}\right]$ increases by roughly $2 \mu \mathrm{S}$ as $f$ goes from 20 to $125 \mathrm{MHz}$ where we use $\nu=2$ at $6.2 \mathrm{~T}$ as the reference point. However, $\operatorname{Re}\left[\sigma_{x x}\right]$ decreases by about 3 $\mu \mathrm{S}$ as $f$ changes from 125 to $400 \mathrm{MHz}$, indicating that $\operatorname{Re}\left[\sigma_{x x}\right]$ vs $f$ has a resonance near $f=125 \mathrm{MHz}$. At P2, an increase of $0.2 \mu \mathrm{S}$ is seen between 20 and $125 \mathrm{MHz}$ and a decrease of $0.2 \mu \mathrm{S}$ occurs between 125 and $400 \mathrm{MHz}$. The variation of $\operatorname{Re}\left[\sigma_{x x}\right]$ with $f$ at $\mathrm{P} 3$, however, is too weak to quantify.

Dependence of $\operatorname{Re}\left[\sigma_{x x}\right]$ on $f$ is also seen close to the IQHE minima in Fig. $2 \mathrm{~b}$ and $2 \mathrm{c}$ at $B=4.43$ and $5.75 \mathrm{~T}$. Detailed discussions of these $f$ dependences can be found in Refs. 13] and 14] and is attributed to the formation of a pinned electron solid in the uppermost partially filled LL near integer $\nu$. 


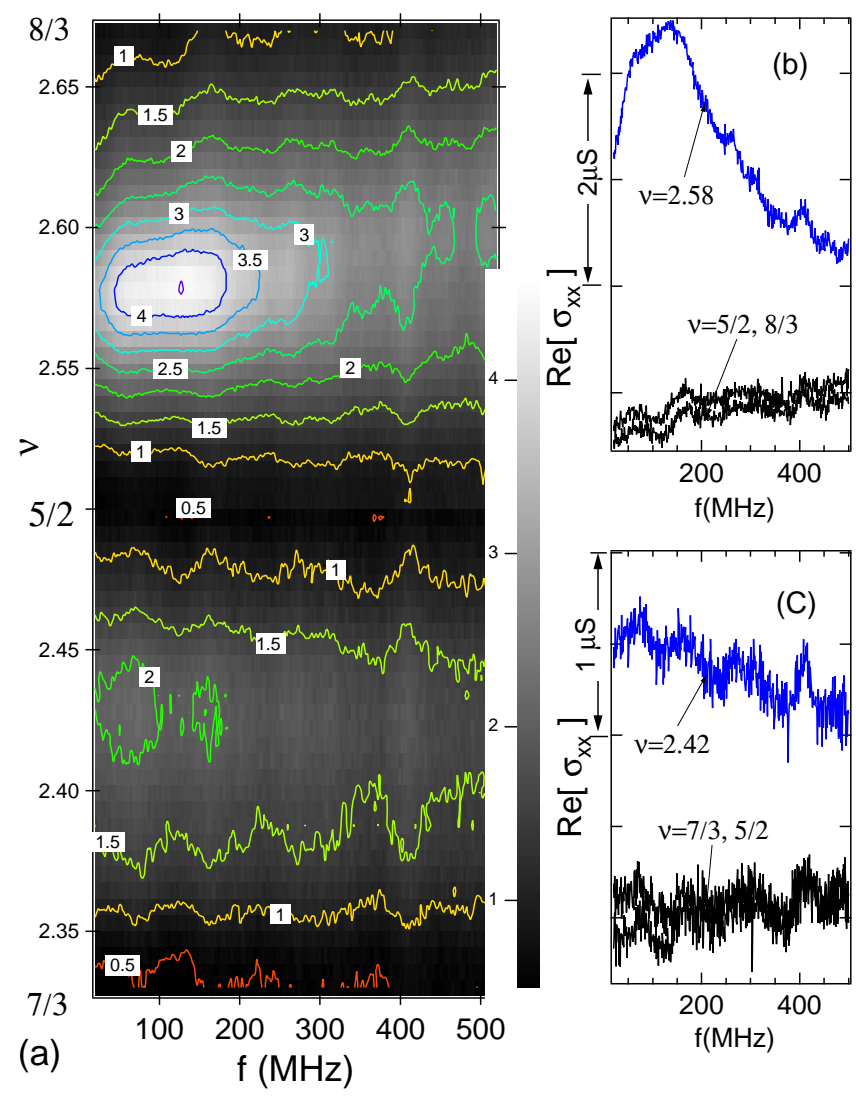

FIG. 3: (a)The real diagonal conductivity, $\operatorname{Re}\left[\sigma_{x x}\right]$ vs frequency $(f)$ in grayscale, between $\nu=7 / 3$ and $8 / 3$ at $T \approx 35$ $\mathrm{mK}$. Contours of constant conductivity are superimposed at intervals of $0.5 \mu \mathrm{S}$. (b) $\operatorname{Re}\left[\sigma_{x x}\right]$ at $\nu=2.58$ compared with $\nu=5 / 2$ and $8 / 3$. (c) $\operatorname{Re}\left[\sigma_{x x}\right]$ at $\nu=2.42$ compared with $\nu=7 / 3$ and $5 / 2$.

In Fig. 3a, we plot $\operatorname{Re}\left[\sigma_{x x}\right]$ in grayscale for $f$ in the 20 to $520 \mathrm{MHz}$ range and $\nu$ between $\nu=7 / 3$ and $8 / 3$. Contours of constant $\operatorname{Re}\left[\sigma_{x x}\right]$ are superimposed at $0.5 \mu \mathrm{S}$ intervals. $\operatorname{Re}\left[\sigma_{x x}\right]$ shows dark bands at the $7 / 3,5 / 2$, and $8 / 3$ FQHE states indicating minima, as expected from the $B$ dependence shown in Fig. 2. Lighter regions in between the FQHE states indicate increased $\operatorname{Re}\left[\sigma_{x x}\right]$. At $\nu \approx 2.58$, a resonance in $\operatorname{Re}\left[\sigma_{x x}\right]$ vs $f$ is seen which peaks at $f \approx 120 \mathrm{MHz}$ and is encircled by the contour lines. This resonance is sharpest and between $\nu=2.57$ ad 2.59, but can be observed for $\nu$ between 2.54 and 2.61. In Fig. $3 \mathrm{~b}$, a spectrum of $\operatorname{Re}\left[\sigma_{x x}\right]$ vs. $f$ at $\nu=2.58$ is shown which exhibits a broad resonance at $f \approx 120 \mathrm{MHz}$. For comparison, $\operatorname{Re}\left[\sigma_{x x}\right]$ spectra taken at $\nu=5 / 2$ and $8 / 3$ are also shown in Fig. $3 \mathrm{~b}$ and are featureless.

In the lower half of Fig. 3a, some increase in $\operatorname{Re}\left[\sigma_{x x}\right]$ is observed between $\nu=7 / 3$ and $5 / 2$. But, the contour lines run nearly parallel to each other as only nonresonant frequency dependence is seen. A weak upturn in $\operatorname{Re}\left[\sigma_{x x}\right]$ is seen between $\nu \approx 2.37$ and $\nu \approx 2.46$ and is most pronounced at $\nu=2.42$. Fig. 3c shows that $\operatorname{Re}\left[\sigma_{x x}\right]$ vs $f$ measured at $\nu=2.42$ exhibits a steady decrease as $f$ increases. This dependence is quite different from the gentle increase in $\operatorname{Re}\left[\sigma_{x x}\right]$ vs $f$ measured at $\nu=7 / 3$ and $5 / 2$. At this sensitive scale, small oscillations are visible in the data which are due to weak reflections between the sample and the room temperature amplifier. The roughly $1 \mu \mathrm{S}$ difference in $\operatorname{Re}\left[\sigma_{x x}\right]$ at $500 \mathrm{MHz}$ is due to the increase in nonresonant background conductivity between the FQHE minima at $7 / 3$ and $5 / 2$ and is also seen in the data in Fig. 2. The error in our determination of $\nu$ in Fig. 3 is \pm 0.01 .

The phenomenology of these N=1 LL RIQHE phases is similar to what has been observed in higher LLs. The dc measurements 20, 21] show that around $\nu^{*}=0.28$, $0.42,0.58$, and 0.70 insulating phases appear in the uppermost partially filled LL for $T \lesssim 50 \mathrm{mK}$. The resonance in $\operatorname{Re}\left[\sigma_{x x}\right]$ shown in Fig. 3b strongly suggests that the insulating phase centered around $\nu=2.58$ is collective in nature - most likely an electron solid - because $f_{p k} \ll k_{B} T / h$. The predictions of theory [22, 23, 24] are that this solid is a two electron per lattice site BP. Therefore, we naturally interpret the resonance we find at $\nu \approx 2.58$ as due to a pinned BP. Furthermore, near $\nu \approx 2.42$, we find an enhancement of $\operatorname{Re}\left[\sigma_{x x}\right]$ at low $f$ which is manifestly different from spectra taken at the $5 / 2$ and $7 / 3$ FQHE states. Rather, the spectra at $\nu=2.42$ is more similar to the resonant behavior at $\nu=2.58$ and hence suggestive of pinned collective insulating behavior.

Recent density matrix renormalization group calculations by Shibata and Yoshioka 22] find a unidirectional charge density wave (stripe) phase for $\nu$ between the $11 / 5$ and $7 / 3$ FQHE states and also between the $7 / 3$ and $5 / 2$ FQHE. Those authors suggest however, that disorder may favor the BP over the stripe phase within these ranges of $\nu$. Mean field theory by Goerbig et al. 23, 24] finds only a $\mathrm{BP}$ in these same ranges of $\nu$ and predicts a transition from the $7 / 3 \mathrm{FQHE}$ to the $\mathrm{BP}$ at $\nu^{*}=0.36$ close to what we observe. The theories [22, 23, 24] invoke particle-hole symmetry for $\nu^{*}=0.5$ to 1 . In contrast, our measurements down to $T=35 \mathrm{mK}$ show that the BP centered around $\nu=2.57$ develops at higher $T$ than the BP centered at $\nu=2.42$. Strangely, this differs from observations of the $\mathrm{BP}$ in $\mathrm{N}=2$ and higher $\mathrm{LL}$, where the electron branch $\left(\nu^{*}<0.5\right)$ always persists to higher $T$ than the hole branch $\left(\nu^{*}>0.5\right)$ of the BP. The development of the $12 / 5$ FQHE state 21] at extremely low $T<25 \mathrm{mK}$ is likely responsible.

In summary, we have studied the $f$ dependence of the RIQHE phases in the $\mathrm{N}=1$ LL between $\nu=2$ and 3 for $T \leq 55 \mathrm{mK}$. We find a resonance in $\operatorname{Re}\left[\sigma_{x x}\right]$ vs $f$ for $\nu=2.58$, where $f_{p k}=120 \pm 15 \mathrm{MHz}$ measured at $35 \mathrm{mK}$. The resonance appears for $\nu$ between 2.54 and 2.61 and is extraordinarily similar to measurements of the $\mathrm{BP}$ in the $\mathrm{N}=2$ and higher LLs 10 . Somewhat sur- 
prisingly, a resonance has not developed at $T \approx 35 \mathrm{mK}$ near $\nu=2.42$, but an upturn in $\operatorname{Re}\left[\sigma_{x x}(f)\right]$ at low $f$ is observed between $\nu=2.37$ and 2.46 at $35 \mathrm{mK}$. The ranges of $\nu$ where these dependences are seen coincide with RIQHE phases reported in Refs. 20] and 21] and with theoretical predictions of the BP 22, 23, 24] in the $\mathrm{N}=1 \mathrm{LL}$. We interpret these data as consistent with formation of an electron solid for $\nu$ between 2.54 and 2.61, presumably a $\mathrm{BP}$, at $T \lesssim 50 \mathrm{mK}$.

We thank Kun Yang and Herb Fertig for stimulating discussions. The measurements were performed at the NHMFL with financial support from the AFOSR and the NHMFL in-house research program. The NHMFL is operated under NSF grant DMR-0084173 with support from the state of Florida.

[1] K. v. Klitzing, G. Dorda, and M. Pepper, Phys. Rev. Lett. 45, 494 (1980).

[2] D. C. Tsui, H. L. Stormer, and A. C. Gossard, Phys. Rev. Lett. 48, 1559 (1982).

[3] M. Lilly et al. , Phys. Rev. Lett. 82, 394 (1999).

[4] R. R. Du et al. Solid State Comm. 109, 389 (1999).

[5] K. B. Cooper et al. Phys. Rev. B 60, R11285 (1999).

[6] M. M. Fogler et al. Phys. Rev. B 54,1853 (1996).

[7] R. Moessner and J. T. Chalker, Phys. Rev. B 54, 5006 (1996).

[8] N. Shibata and D. Yoshioka, Phys. Rev. Lett. 86, 5755 (2001).

[9] F. H. D. Haldane, E. H. Rezayi, and Kun Yang, Phys. Rev. Lett. 86, 5755 (2001).

[10] R.M. Lewis, et al. Phys. Rev. Lett. 89136804 (2002).
[11] K. B. Cooper et al. Phys. Rev. Lett. 90, 226803 (2003).

[12] R. M. Lewis, Yong Chen, L. W. Engel, D. C. Tsui, P. D. Ye, L. N. Pfeiffer, and K. W. West, Phys. Rev. Lett. 93,

[13] Yong Chen, R. M. Lewis, L. W. Engel, D. C. Tsui, P. D. Ye, L. N. Pfeiffer, and K. W. West, Phys. Rev. Lett. 91, 016801(2003).

[14] R. M. Lewis, Yong P. Chen, L. W. Engel, D. C. Tsui, P. D. Ye, L. N. Pfeiffer, and K. W. West, Physica E 22, 104 (2004).

[15] for reviews of the Wigner crystal see chapter 3 by $\mathrm{H}$. Fertig and chapter 9 by M. Shayegan of Perspectives in Quantum Hall Physics, edited by S. Das Sarma and A. Pinzcuk (Wiley and Sons, New York, 1997).

[16] P. D. Ye et al. Phys. Rev. Lett. 89176802 (2002).

[17] L. W. Engel et al. Solid State Commun. 104, 167 (1997).

[18] H. Fukuyama and P. A. Lee, Phys. Rev. B 18, 6245 (1978).

[19] R. Chitra, T. Giamarchi, and P. Le Doussal, Phys. Rev. B 65, 035312(2001).

[20] J. P. Eisenstein, K. B. Cooper, L. N. Pfeiffer, and K. W. West, Phys. Rev. Lett. 88, 076801 (2002).

[21] J. S. Xia, W. Pan, C. L. Vicente, E. D. Adams, N. S. Sullivan, H. L. Stormer, D. C. Tsui, L. N. Pfeiffer, K. W. Baldwin, and K. W. West, Phys. Rev. Lett. 93, 176809 (2004).

[22] N. Shibata and D. Yoshioka, J. Phys. Soc. Jpn. 72, 664 (2003).

[23] M. O, Goerbig, P. Lederer, and C. Morais Smith, Phys. Rev. B 68, R241302 (2003).

[24] M. O. Goerbig, P. Lederer, and C. Morais Smith, Phys. Rev. B 69, 115327 (2004).

[25] C. P. Wen, IEEE Trans. Microwave Theory Tech. 17, 1087 (1969).

[26] L. W. Engel et al. Phys. Rev. Lett. 71, 2638 (1993). 


\title{
Microwave resonance of the reentrant insulating quantum Hall phases in the 1st excited Landau Level
}

\author{
R. M. Lewis ${ }^{1,2}$, Yong P. Chen ${ }^{1,2}$, L. W. Engel ${ }^{1}$, D. C. Tsui ${ }^{2}$, L. N. Pfeiffer ${ }^{3}$, and K. W. West ${ }^{3}$ \\ ${ }^{1}$ NHMFL, Florida State University, Tallahassee, FL 32310, USA \\ ${ }^{2}$ Dept. of Electrical Engineering, Princeton University, Princeton, NJ 08544 \\ ${ }^{3}$ Bell Laboratories, Lucent Technologies, Murray Hill, NJ 07974
}

(Dated: November 25, 2018)

\begin{abstract}
We present measurements of the real diagonal microwave conductivity of the reentrant insulating quantum Hall phases in the first excited Landau level at temperatures below $50 \mathrm{mK}$. A resonance is detected around filling factor $\nu=2.58$ and weaker frequency dependence is seen at $\nu=2.42$ and 2.28. These measurements are consistent with the formation of a bubble phase crystal centered around these $\nu$ at very low temperatures.
\end{abstract}

Clean two-dimensional electron systems (2DES) show a plethora of phases at low temperatures $(T)$ when subjected to perpendicular magnetic fields $(B)$. The most well known of these are the integer [1] and fractional 2] quantum Hall effects (IQHE and FQHE). Both are identified by quantized Hall resistances, $R_{x y}=h / \nu e^{2}$ and the simultaneous vanishing of the diagonal resistance $\left(R_{x x}\right)$ when the filling factor $\nu=n h / e B$ coincides with a gap in the single or many-particle density of states. In higher Landau levels (LL), $N \geq 2$, where $N$ is the LL index, vanishing minima in $R_{x x}$ are observed [3, 4] near $\nu=4+1 / 4,4+3 / 4$, and several subsequent odd quarter $\nu$. However, at these $\nu, R_{x y}$ is quantized to the value of the adjacent integer plateau. Therefore these states are said to exhibit a reentrant integer quantum Hall effect (RIQHE) [5]. Theory [6, 7, [8, [9] predicts a crystal phase of the 2DES with triangular lattice symmetry and two or more electrons per lattice site - pinned by disorder and hence insulating - to occur at approximately these $\nu$. This crystal has been dubbed the bubble phase (BP). Experiments have subsequently shown nonlinear current-voltage characteristics 5, a microwave resonance [10], and narrow band noise [11], in the range of $\nu$ centered around $\nu=4+1 / 4$. Still more recent work 12 has looked at the transition between the BP around $\nu=4+1 / 4$ and another solid phase - presumably a Wigner crystal [13, 14, 15] of carriers in the uppermost LL-close to $\nu=4$.

The microwave measurements 10, 12], which are similar to those we will discuss here, provide compelling evidence that the RIQHE is due to the formation of a pinned electron solid such as the BP. These measurements detect a sharp resonance in the real diagonal conductivity $\left(\operatorname{Re}\left[\sigma_{x x}(f)\right]\right)$ when the $2 \mathrm{DES}$ forms an electron solid. The observed resonant frequency, $f_{p k}$, is considerably lower, in terms of energy, than the temperature at which the resonance first appears, $f_{p k}<<k_{B} T / h$, indicating that the resonance is not due to the ionization of individual carriers trapped in potential defects. Furthermore, this resonance is strikingly similar to the resonance which occurs in the insulating phase of $2 \mathrm{DES}[16,17$ that terminates the FQHE at high $B, \nu<1 / 5$, where the 2 DES is thought to form a Wigner crystal[15]. The resonances in both regimes (RIQHE and $\nu<1 / 5$ insulating phase) are interpreted as pinning modes 18, 19] of crystal domains oscillating within the disorder potential.

Recently, Eisenstein et al. 20] and Xia et al. 21] have presented measurements of RIQHE's in the $N=1$ LL which appear only at $T \lesssim 50 \mathrm{mK}$. These RIQHE's are centered at partial filling factors $\nu^{*} \approx 0.28,0.42$, 0.56 , and 0.70 where $\nu^{*}=\nu-[\nu]$ and $[\nu]$ is the greatest integer less than $\nu$. The initial theories [6, 7] of charge density waves in higher LL (which predicted the stripe and $\mathrm{BP}$ for $\nu>4$ ) did not consider the $\mathrm{N}=1 \mathrm{LL}$. Also, the short range softening of the effective electron-electron potential, which gives rise to the BP in higher LL, is only weakly present in the $\mathrm{N}=1 \mathrm{LL}$ and absent in the $\mathrm{N}=0 \mathrm{LL}$. However, more recent theories 22, 23, 24] do predict a BP and perhaps a stripe phase in the first excited LL.

In this paper, we present measurements of $\operatorname{Re}\left[\sigma_{x x}\right]$ between $\nu=2$ and 3 . At $T \approx 35 \mathrm{mK}$, we show that a resonance occurs in the frequency $(f)$ dependence of $\operatorname{Re}\left[\sigma_{x x}\right]$ in a range of $\nu$ around 2.58. An enhancement of $\operatorname{Re}\left[\sigma_{x x}\right]$ at $\nu \approx 2.42$ is also observed at low $f$. These features are not present for $T \geq 55 \mathrm{mK}$. The resonance occurs at frequency, $f \sim 125 \mathrm{MHz}$, and is coincident with the RIQHE phase found previously [20, 21] in the $\mathrm{N}=1 \mathrm{LL}$ on either side of $\nu=5 / 2$. In light of our earlier observations 10 . in higher LL's, this resonance is naturally interpreted as due to the pinning mode of the $\mathrm{BP}$ in the $\mathrm{N}=1 \mathrm{LL}$ at $\nu=2.57$.

The sample, grown by molecular beam epitaxy, is a $300 \AA \mathrm{GaAs} / \mathrm{AlGaAs}$ quantum well of density $\mathrm{n}=3.0 \times 10^{11} \mathrm{~cm}^{-2}$ and mobility $\mu=2.4 \times$ $10^{7} \mathrm{~cm}^{2} \mathrm{~V}^{-1} \mathrm{~s}^{-1}$ at $300 \mathrm{mK}$. The quantum well is approximately $2000 \AA$ below the surface. A coplanar waveguide [25] (CPW) is patterned onto the sample surface. The CPW consists of a driven centerline of length $l$ which is separated from symmetrically placed ground planes by slots of width $w$. At high $f$ and small $\left|\sigma_{x x}\right|$, the conductivity of the 2DES attenuates power transmission along the $\mathrm{CPW}$ and $\operatorname{Re}\left[\sigma_{x x}\right]=-\frac{w}{2 l Z_{0}} \ln \left(P_{t} / P_{0}\right)$, where $P_{t}$ is the transmitted power 26]. In the absence of 2DES, the CPW has impedance $Z_{0}=50 \Omega$ and transmits power $P_{0}$. 


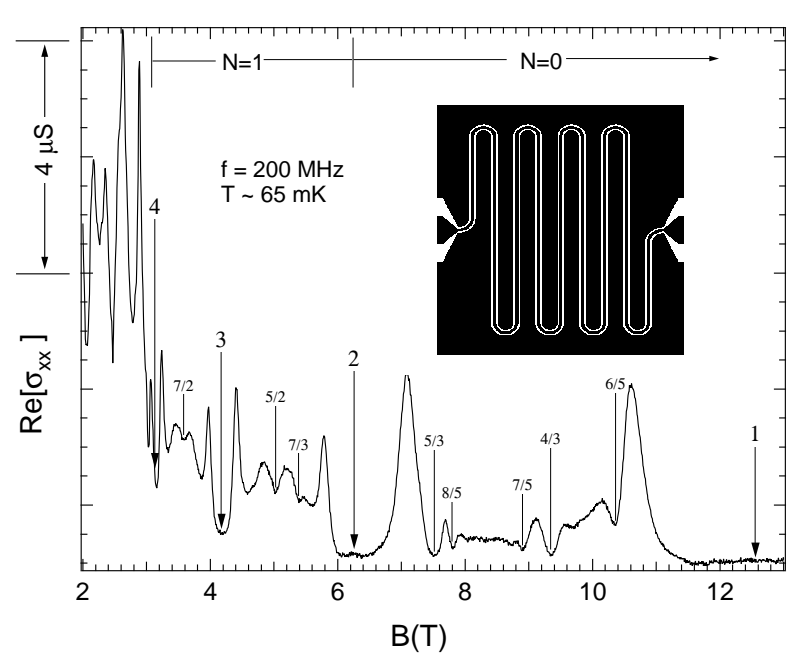

FIG. 1: The real part of the diagonal conductivity, $\operatorname{Re}\left[\sigma_{x x}\right]$ versus $B$ between 2 and $13 \mathrm{~T}$. Inset is the coplanar waveguide pattern in which dark regions are the metal films.

The CPW pattern is shown in the inset to Fig. 1 and has $l=28 \mathrm{~mm}$ and $w=30 \mu \mathrm{m}$. The dark regions are the metal films of the CPW. Measurements were carried out in the low power limit which was determined by reducing microwave power until no further change in $\operatorname{Re}\left[\sigma_{x x}\right]$ was observed.

In Fig. 1 we show $\operatorname{Re}\left[\sigma_{x x}\right]$ measured, between 2 and $13 \mathrm{~T}$ at $200 \mathrm{MHz}$ and $T \approx 65 \mathrm{mK}$. The broad minima centered around $B=12.55,6.26,4.18$, and $3.14 \mathrm{~T}$ are due to the IQHE states at $\nu=1,2,3$, and 4 . Between $\nu=1$ and 2, sharp minima indicate FQHE states in the $\mathrm{N}=0$ LL at $\nu=6 / 5,4 / 3,7 / 5,8 / 5$, and $5 / 3$. The $\mathrm{N}=1$ LL also shows FQHE states. For instance, at $B=5.38$, 5.03 , and $3.58 \mathrm{~T}$, dips due to the $\nu=7 / 3$, the $5 / 2$, and $7 / 2$ FQHE are apparent. For $\nu>4(\mathrm{~N}=2 \mathrm{LL})$, sharp peaks appear in $\operatorname{Re}\left[\sigma_{x x}\right]$ at $B \approx 2.89,2.63,2.36$, and $2.17 \mathrm{~T}$, or roughly at $\nu \approx 4+1 / 4,4+3 / 4,5+1 / 4$, and $5+3 / 4$ where DC measurements [3, 4, 5] observe the RIQHE. These peaks are due to a resonance at $f \approx 300$ $\mathrm{MHz}$, which was interpreted [10] as due to the pinning mode 18 of the BP.

In Fig. 2, we focus on the range of $B$ between 4.2 and $6.2 \mathrm{~T}$ where $\nu$ lies within the $\mathrm{N}=1$ spin-up LL. In Fig. 2a, we show $\operatorname{Re}\left[\sigma_{x x}\right]$ at $f=125 \mathrm{MHz}$ for $T$ approximately at $30,40,50$ and $55 \mathrm{mK}$. The most striking aspects of these data are the dramatic increases of the peaks in $\operatorname{Re}\left[\sigma_{x x}\right]$ at $4.88 \mathrm{~T}$ (marked as P1) and at $5.17 \mathrm{~T}$ (marked as P2) with decreasing $T$. P1 occurs at $\nu=2.57$ and $\mathrm{P} 2$ at $\nu=2.42$. At $\mathrm{P} 1, \operatorname{Re}\left[\sigma_{x x}\right]$ increases by $\sim 4 \mu \mathrm{S}$ as $T$ decreases from $55 \mathrm{mK}$ to $30 \mathrm{mK}$. Similarly, at $\mathrm{P} 2, \operatorname{Re}\left[\sigma_{x x}\right]$ increases by about $1 \mu \mathrm{S}$ over the same $T$ range. Some $T$ dependence is also evident in the peak at $B=5.47 \mathrm{~T}(\nu=2.28)$, labelled as P3.

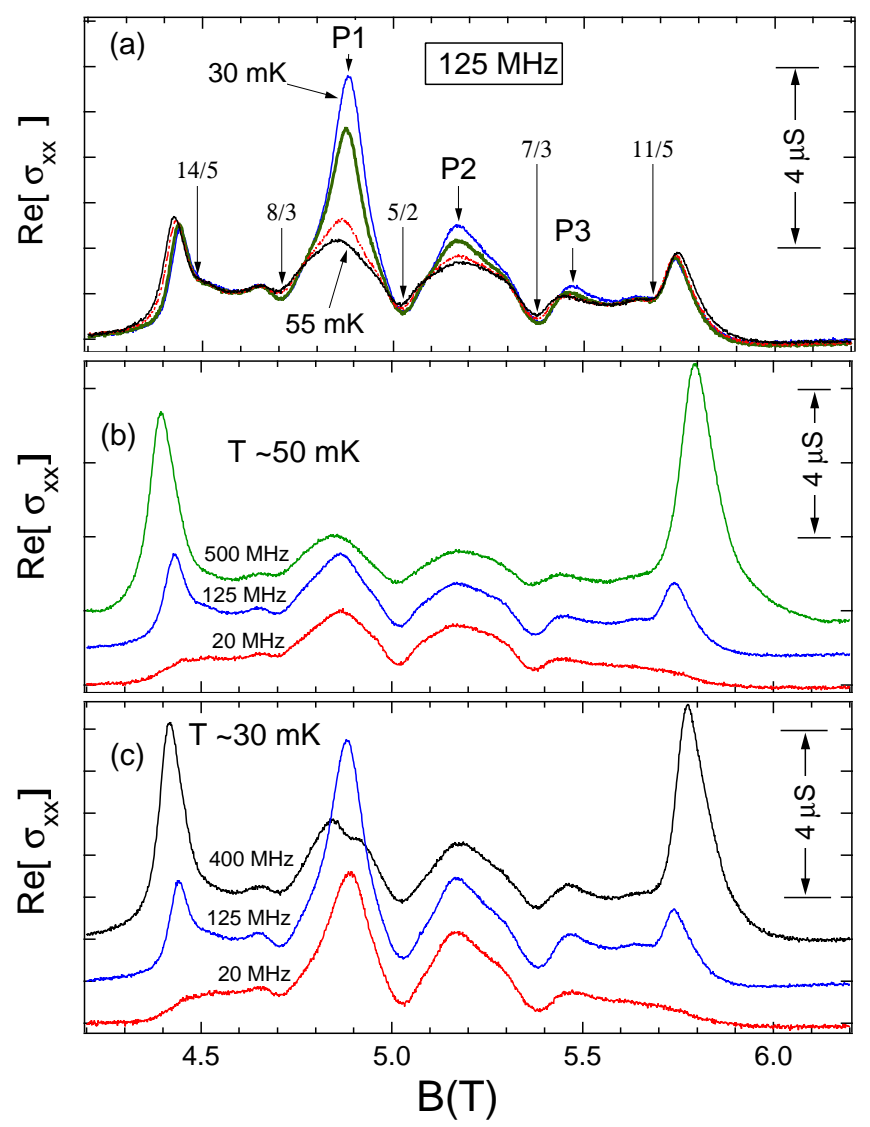

FIG. 2: (a) $\operatorname{Re}\left[\sigma_{x x}\right]$ vs $B$ between 4.2 and $6.2 \mathrm{~T}$ at frequency $f=125 \mathrm{MHz}$ for temperatures $T \approx 30,40,50$, and $55 \mathrm{mK}$. (b) $\operatorname{Re}\left[\sigma_{x x}\right]$ vs $B$ at $f=20,125$, and $500 \mathrm{MHz}$ with $T \approx 50$ mK offset $1 \mu \mathrm{S}$ from each other. (c), $\operatorname{Re}\left[\sigma_{x x}\right]$ vs $B$ at $f=20$, 125 , and $400 \mathrm{MHz}$, all at $T=30 \mathrm{mK}$ offset for clarity.

Fig. $2 \mathrm{~b}$ shows $\operatorname{Re}\left[\sigma_{x x}\right]$ vs $B$ measured at $f=20,125$, and $500 \mathrm{MHz}$ at $T \approx 50 \mathrm{mK}$. At this $T, \mathrm{P} 1$ and $\mathrm{P} 2$, show little dependence on $f$. The traces are offset $1 \mu \mathrm{S}$ from each other.

In Fig. 2c, we plot $\operatorname{Re}\left[\sigma_{x x}\right]$ vs $B$ for $f=20,125$, and $400 \mathrm{MHz}$, at $T \approx 30 \mathrm{mK}$ offset by $1 \mu \mathrm{S}$ each for clarity. At $\mathrm{P} 1, \operatorname{Re}\left[\sigma_{x x}\right]$ increases by roughly $2 \mu \mathrm{S}$ as $f$ goes from 20 to $125 \mathrm{MHz}$ where we use $\nu=2$ at $6.2 \mathrm{~T}$ as the reference point. However, $\operatorname{Re}\left[\sigma_{x x}\right]$ decreases by about 3 $\mu \mathrm{S}$ as $f$ changes from 125 to $400 \mathrm{MHz}$, indicating that $\operatorname{Re}\left[\sigma_{x x}\right]$ vs $f$ has a resonance near $f=125 \mathrm{MHz}$. At P2, an increase of $0.2 \mu \mathrm{S}$ is seen between 20 and $125 \mathrm{MHz}$ and a decrease of $0.2 \mu \mathrm{S}$ occurs between 125 and $400 \mathrm{MHz}$. The variation of $\operatorname{Re}\left[\sigma_{x x}\right]$ with $f$ at $\mathrm{P} 3$, however, is too weak to quantify.

Dependence of $\operatorname{Re}\left[\sigma_{x x}\right]$ on $f$ is also seen close to the IQHE minima in Fig. $2 \mathrm{~b}$ and $2 \mathrm{c}$ at $B=4.43$ and $5.75 \mathrm{~T}$. Detailed discussions of these $f$ dependences can be found in Refs. 13] and 14] and is attributed to the formation of a pinned electron solid in the uppermost partially filled 


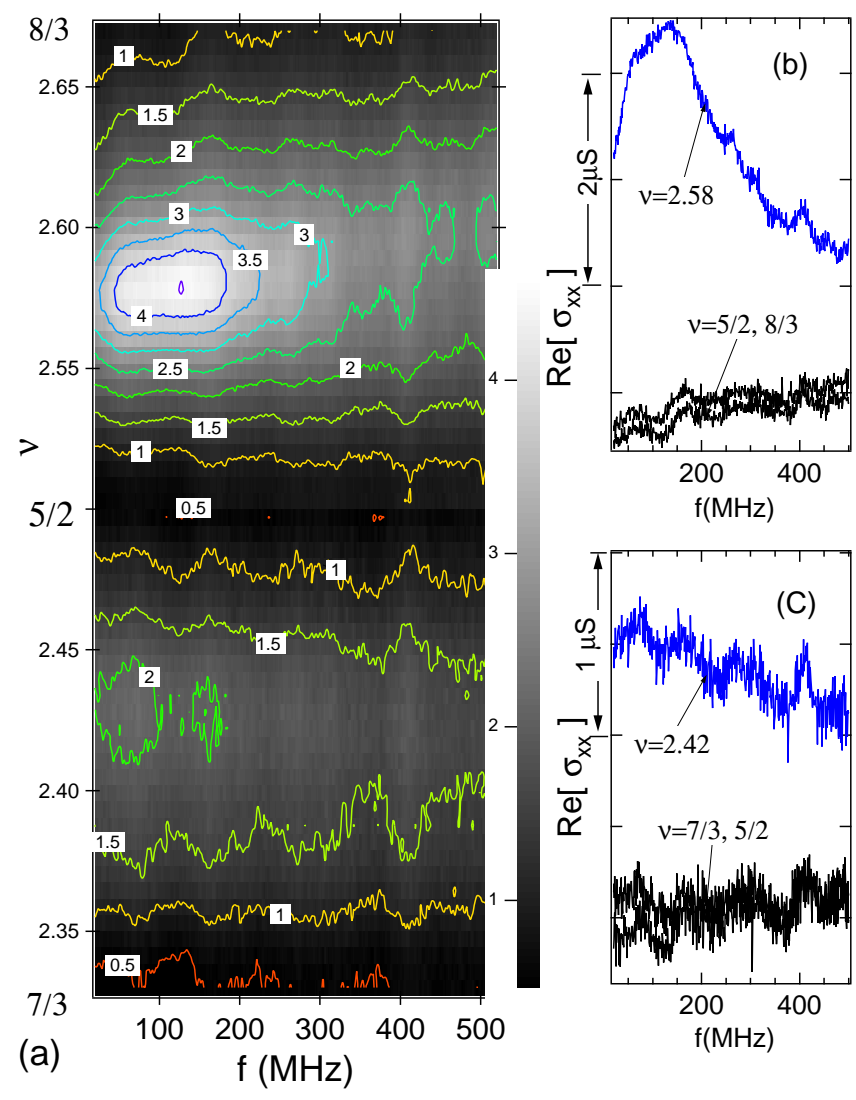

FIG. 3: (a)The real diagonal conductivity, $\operatorname{Re}\left[\sigma_{x x}\right]$ vs frequency $(f)$ in grayscale, between $\nu=7 / 3$ and $8 / 3$ at $T \approx 35$ $\mathrm{mK}$. Contours of constant conductivity are superimposed at intervals of $0.5 \mu \mathrm{S}$. (b) $\operatorname{Re}\left[\sigma_{x x}\right]$ at $\nu=2.58$ compared with $\nu=5 / 2$ and $8 / 3$. (c) $\operatorname{Re}\left[\sigma_{x x}\right]$ at $\nu=2.42$ compared with $\nu=7 / 3$ and $5 / 2$.

\section{LL near integer $\nu$.}

In Fig. 3a, we plot $\operatorname{Re}\left[\sigma_{x x}\right]$ in grayscale for $f$ in the 20 to $520 \mathrm{MHz}$ range and $\nu$ between $\nu=7 / 3$ and $8 / 3$. Contours of constant $\operatorname{Re}\left[\sigma_{x x}\right]$ are superimposed at $0.5 \mu \mathrm{S}$ intervals. $\operatorname{Re}\left[\sigma_{x x}\right]$ shows dark bands at the $7 / 3,5 / 2$, and 8/3 FQHE states indicating minima, as expected from the $B$ dependence shown in Fig. 2. Lighter regions in between the FQHE states indicate increased $\operatorname{Re}\left[\sigma_{x x}\right]$. At $\nu \approx 2.58$, a resonance in $\operatorname{Re}\left[\sigma_{x x}\right]$ vs $f$ is seen which peaks atf $\approx 120 \mathrm{MHz}$ and is encircled by the contour lines. This resonance is sharpest and between $\nu=2.57$ ad 2.59, but can be observed for $\nu$ between 2.54 and 2.61. In Fig. $3 \mathrm{~b}$, a spectrum of $\operatorname{Re}\left[\sigma_{x x}\right]$ vs. $f$ at $\nu=2.58$ is shown which exhibits a broad resonance at $f \approx 120 \mathrm{MHz}$. For comparison, $\operatorname{Re}\left[\sigma_{x x}\right]$ spectra taken at $\nu=5 / 2$ and $8 / 3$ are also shown in Fig. $3 \mathrm{~b}$ and are featureless.

In the lower half of Fig. 3a, some increase in $\operatorname{Re}\left[\sigma_{x x}\right]$ is observed between $\nu=7 / 3$ and $5 / 2$. But, the contour lines run nearly parallel to each other as only nonresonant frequency dependence is seen. A weak upturn in $\operatorname{Re}\left[\sigma_{x x}\right]$ is seen between $\nu \approx 2.37$ and $\nu \approx 2.46$ and is most pronounced at $\nu=2.42$. Fig. $3 \mathrm{c}$ shows that $\operatorname{Re}\left[\sigma_{x x}\right]$ vs $f$ measured at $\nu=2.42$ exhibits a steady decrease as $f$ increases. This dependence is quite different from the gentle increase in $\operatorname{Re}\left[\sigma_{x x}\right]$ vs $f$ measured at $\nu=7 / 3$ and $5 / 2$. At this sensitive scale, small oscillations are visible in the data which are due to weak reflections between the sample and the room temperature amplifier. The roughly $1 \mu \mathrm{S}$ difference in $\operatorname{Re}\left[\sigma_{x x}\right]$ at $500 \mathrm{MHz}$ is due to the increase in nonresonant background conductivity between the FQHE minima at $7 / 3$ and $5 / 2$ and is also seen in the data in Fig. 2. The error in our determination of $\nu$ in Fig. 3 is \pm 0.01 .

The phenomenology of these $\mathrm{N}=1$ LL RIQHE phases is similar to what has been observed in higher LLs. The dc measurements [20, 21] show that around $\nu^{*}=0.28$, $0.42,0.58$, and 0.70 insulating phases appear in the uppermost partially filled LL for $T \lesssim 50 \mathrm{mK}$. The resonance in $\operatorname{Re}\left[\sigma_{x x}\right]$ shown in Fig. 3b strongly suggests that the insulating phase centered around $\nu=2.58$ is collective in nature - most likely an electron solid-because $f_{p k} \ll k_{B} T / h$. The predictions of theory [22, 23, 24] are that this solid is a two electron per lattice site BP. Therefore, we naturally interpret the resonance we find at $\nu \approx 2.58$ as due to a pinned BP. Furthermore, near $\nu \approx 2.42$, we find an enhancement of $\operatorname{Re}\left[\sigma_{x x}\right]$ at low $f$ which is manifestly different from spectra taken at the $5 / 2$ and $7 / 3$ FQHE states. Rather, the spectra at $\nu=2.42$ is more similar to the resonant behavior at $\nu=2.58$ and hence suggestive of pinned collective insulating behavior.

Recent density matrix renormalization group calculations by Shibata and Yoshioka 22] find a unidirectional charge density wave (stripe) phase for $\nu$ between the $11 / 5$ and $7 / 3$ FQHE states and also between the $7 / 3$ and $5 / 2$ FQHE. Those authors suggest however, that disorder may favor the BP over the stripe phase within these ranges of $\nu$. Mean field theory by Goerbig et al. [23, 24] finds only a $\mathrm{BP}$ in these same ranges of $\nu$ and predicts a transition from the $7 / 3 \mathrm{FQHE}$ to the $\mathrm{BP}$ at $\nu^{*}=0.36$ close to what we observe. The theories [22, 23, 24] invoke particle-hole symmetry for $\nu^{*}=0.5$ to 1 . In contrast, our measurements down to $T=35 \mathrm{mK}$ show that the $\mathrm{BP}$ centered around $\nu=2.57$ develops at higher $T$ than the BP centered at $\nu=2.42$. Strangely, this differs from observations of the $\mathrm{BP}$ in $\mathrm{N}=2$ and higher LL, where the electron branch $\left(\nu^{*}<0.5\right)$ always persists to higher $T$ than the hole branch $\left(\nu^{*}>0.5\right)$ of the BP. The development of the $12 / 5$ FQHE state 21] at extremely low $T<25 \mathrm{mK}$ is likely responsible.

In summary, we have studied the $f$ dependence of the RIQHE phases in the $\mathrm{N}=1$ LL between $\nu=2$ and 3 for $T \leq 55 \mathrm{mK}$. We find a resonance in $\operatorname{Re}\left[\sigma_{x x}\right]$ vs $f$ for $\nu=2.58$, where $f_{p k}=120 \pm 15 \mathrm{MHz}$ measured at $35 \mathrm{mK}$. The resonance appears for $\nu$ between 2.54 and 2.61 and is extraordinarily similar to measurements of 
the $\mathrm{BP}$ in the $\mathrm{N}=2$ and higher LLs 10 . Somewhat surprisingly, a resonance has not developed at $T \approx 35 \mathrm{mK}$ near $\nu=2.42$, but an upturn in $\operatorname{Re}\left[\sigma_{x x}(f)\right]$ at low $f$ is observed between $\nu=2.37$ and 2.46 at $35 \mathrm{mK}$. The ranges of $\nu$ where these dependences are seen coincide with RIQHE phases reported in Refs. 20] and 21] and with theoretical predictions of the BP 22, 23, 24] in the $\mathrm{N}=1 \mathrm{LL}$. We interpret these data as consistent with formation of an electron solid for $\nu$ between 2.54 and 2.61, presumably a $\mathrm{BP}$, at $T \lesssim 50 \mathrm{mK}$.

We thank Kun Yang and Herb Fertig for stimulating discussions. The measurements were performed at the NHMFL with financial support from the AFOSR and the NHMFL in-house research program. The NHMFL is operated under NSF grant DMR-0084173 with support from the state of Florida.

[1] K. v. Klitzing, G. Dorda, and M. Pepper, Phys. Rev. Lett. 45, 494 (1980).

[2] D. C. Tsui, H. L. Stormer, and A. C. Gossard, Phys. Rev. Lett. 48, 1559 (1982).

[3] M. Lilly et al. , Phys. Rev. Lett. 82, 394 (1999).

[4] R. R. Du et al. Solid State Comm. 109, 389 (1999).

[5] K. B. Cooper et al. Phys. Rev. B 60, R11285 (1999).

[6] M. M. Fogler et al. Phys. Rev. B 54,1853 (1996).

[7] R. Moessner and J. T. Chalker, Phys. Rev. B 54, 5006 (1996).

[8] N. Shibata and D. Yoshioka, Phys. Rev. Lett. 86, 5755 (2001).

[9] F. H. D. Haldane, E. H. Rezayi, and Kun Yang, Phys. Rev. Lett. 86, 5755 (2001).
[10] R.M. Lewis, et al. Phys. Rev. Lett. 89136804 (2002).

[11] K. B. Cooper et al. Phys. Rev. Lett. 90, 226803 (2003).

[12] R. M. Lewis, Yong Chen, L. W. Engel, D. C. Tsui, P. D. Ye, L. N. Pfeiffer, and K. W. West, Phys. Rev. Lett. 93,

[13] Yong Chen, R. M. Lewis, L. W. Engel, D. C. Tsui, P. D. Ye, L. N. Pfeiffer, and K. W. West, Phys. Rev. Lett. 91, 016801(2003).

[14] R. M. Lewis, Yong P. Chen, L. W. Engel, D. C. Tsui, P. D. Ye, L. N. Pfeiffer, and K. W. West, Physica E 22, 104 (2004).

[15] for reviews of the Wigner crystal see chapter 3 by $\mathrm{H}$. Fertig and chapter 9 by M. Shayegan of Perspectives in Quantum Hall Physics, edited by S. Das Sarma and A. Pinzcuk (Wiley and Sons, New York, 1997).

[16] P. D. Ye et al. Phys. Rev. Lett. 89176802 (2002).

[17] L. W. Engel et al. Solid State Commun. 104, 167 (1997).

[18] H. Fukuyama and P. A. Lee, Phys. Rev. B 18, 6245 (1978).

[19] R. Chitra, T. Giamarchi, and P. Le Doussal, Phys. Rev. B 65, 035312(2001).

[20] J. P. Eisenstein, K. B. Cooper, L. N. Pfeiffer, and K. W. West, Phys. Rev. Lett. 88, 076801 (2002).

[21] J. S. Xia, W. Pan, C. L. Vicente, E. D. Adams, N. S. Sullivan, H. L. Stormer, D. C. Tsui, L. N. Pfeiffer, K. W. Baldwin, and K. W. West, Phys. Rev. Lett. 93, 176809 (2004).

[22] N. Shibata and D. Yoshioka, J. Phys. Soc. Jpn. 72, 664 (2003).

[23] M. O, Goerbig, P. Lederer, and C. Morais Smith, Phys. Rev. B 68, R241302 (2003).

[24] M. O. Goerbig, P. Lederer, and C. Morais Smith, Phys. Rev. B 69, 115327 (2004).

[25] C. P. Wen, IEEE Trans. Microwave Theory Tech. 17, 1087 (1969).

[26] L. W. Engel et al. Phys. Rev. Lett. 71, 2638 (1993). 